\title{
Treatment of advanced solid tumours with NSAIDs: Correlation of quantitative monitoring of circulating tumour cells and positron emission tomography-computed tomography imaging
}

\author{
REGINA WILLECKE-HOCHMUTH ${ }^{1}$, KATHARINA PACHMANN $^{1}$ and JOACHIM DREVS ${ }^{2}$ \\ ${ }^{1}$ Transfusion Medical Centre Bayreuth, D-95448 Bayreuth; ${ }^{2}$ UNIFONTIS Clinic, D-72076 Tübingen, Germany
}

Received October 28, 2015; Accepted June 7, 2016

DOI: $10.3892 / \mathrm{ol} .2016 .4878$

\begin{abstract}
The detection and characterisation of tumour-derived circulating epithelial tumor cells (CETCs) or circulating tumor cells (CTCs) have been a main focus of basic oncological research over previous years. Numerous studies in the past decade have shown that CTCs are a promising tool for the estimation of the risk for metastatic relapse. The present observational study describes treatment results using tumour imaging and the quantification of CTCs. A group of 14 patients with advanced carcinomas was followed during their anticancer treatments. CTC numbers were serially detected and treatment success was estimated by positron emission tomography-computed tomography. A connection was found between tumour remission and a decreasing CTC count in $83 \%$, a connection between stable disease and stable CTC numbers in $78 \%$ and a connection between progressive disease (PD) and an increase in CTC count in 50\% of cases. In the patients with $\mathrm{PD}$, an incomplete response was observed affecting the CTCs, but not the solid region of the tumour. As a result of this study, it may be concluded that patients with solid tumours benefit from serial quantification of CTCs in addition to imaging, as this combination of techniques provides a more sensitive result than imaging alone.
\end{abstract}

\section{Introduction}

Non-steroidal anti-inflammatory drugs (NSAIDs) have been used for a long time to treat rheumatic diseases and the signs and symptoms of inflammation. Additionally, NSAIDs have been discussed in the last decade as potential anticancer agents $(1,2)$. After the initial observation that NSAIDs could inhibit tumour growth in the lungs and intestines of rodents,

Correspondence to: Dr Regina Willecke-Hochmuth, Transfusion Medical Centre Bayreuth, Kurpromenade 2, D-95448 Bayreuth, Germany

E-mail: rwillecke@laborpachmann.de

Key words: non-steroidal anti-inflammatory drug, circulating tumour cells, positron emission tomography-computed tomography, solid tumours, diflunisal, salicylates, chemosensitivity testing it was confirmed that there is a substantial risk reduction for colon cancer when patients regularly take acetylsalicylic acid (ASA). Furthermore, evidence is accumulating that the use of NSAIDs confers a protective effect against gastric and oesophageal cancer, and maybe also against prostatic, ovarian and lung cancer (3). In 2012, a retrospective study showed that the use of the NSAID ketorolac in breast cancer patients was associated with a superior disease-free survival rate in the first years after surgery (4).

The NSAID treatment described in the present study follows the recommendations of Kreutz on how to apply the drugs diflunisal, ASA and 4-aminosalicylic acid (PAS) to treat cancer (5). The aforementioned salicylates show a cytotoxic effect on cancer cell lines in vitro when used in physiologic concentrations. The mechanism of action is proposed to be $\mathrm{NF}-\kappa \mathrm{B}$ inhibition leading to cell cycle arrest and subsequent apoptosis (6). The anti-inflammatory effect of NSAIDs could also inhibit cancer progression and metastasis formation, as inflammation is hypothesised to be a possible cause $(3,7,8)$. The first clinical data indicate that patients with advanced solid tumours benefit from salicylate therapy (Drevs, unpublished data).

In the present study, the beneficial effect of the NSAID treatment was closely examined by positron emission tomography-computed tomography (PET-CT). The latter, however, does not always give clear results and is an expensive method. Therefore, the effect of the therapy on the systemic region of the tumour, the circulating tumour cells (CTCs), was simultaneously monitored using an approach that aimed to encompass all of these cells in the blood without loss due to enrichment procedures and could be performed repeatedly. The detection and characterisation of tumour-derived circulating epithelial tumour cells (CETCs) has been a main focus of basic oncologic research in previous years. CTCs disseminate from solid tumours, circulate in the blood or lymphatic system and are claimed to be the cause of distant metastases (9-11). In 2006, a direct comparison between the enumeration of CTCs and radiological imaging in metastatic breast cancer patients for the prediction of overall survival was published for the first time. The study showed that CTC enumeration is a reliable way to monitor disease progression (12). However, the isolation procedure in the study was accompanied by a massive loss of CTCs, as the threshold was only 5 CTCs. A method that leads to a higher yield, the analysis of CTCs and their course by the 
maintrac ${ }^{\circledR}$ approach has been shown to correlate with clinical outcome in breast cancer patients and provides a novel analytic tool, which is probably an alternative to invasive biopsies. The reduction or marginal changes of CTC numbers during chemotherapy corresponds with a good prognosis, whereas an increase corresponds with a higher risk of metastases (13-15). The present study shows that the dissemination of CTCs from an epithelial tumour or from metastases can be monitored over time to assess the response to a treatment.

\section{Patients and methods}

Patient population. Clinical data was collected from 14 patients (mean age, 55.5 years) with advanced and heavily pre-treated epithelial tumours who received an anti-cancer treatment in the UNIFONTIS clinic (Tübingen, Germany) and who were followed up by PET-CT, with additional monitoring of CTC numbers in the blood (Table I). The majority of the patients $(57 \%)$ underwent primary tumour removal. The remaining $43 \%$ of patients did not undergo primary tumor removal due to unresectable tumors or patient refusal. A total of $93 \%$ had histologically confirmed distant metastases at the time of treatment.

Salicylate therapy. A total of 10 patients underwent treatment with salicylate therapy. The treatment with diflunisal or other salicylates (ASA and PAS) was applied following the recommendations of Kreutz (5). Usually, the drugs were administered intravenously for 4 days a week, 2 weeks in a row. The initial dose of salicylates on the first day was $35 \mathrm{mg} / \mathrm{kg}$, administered intravenously. On the second to fourth days, patients received a dose of $30 \mathrm{mg} / \mathrm{kg}$, administered intravenously. Intravenous application was selected to avoid gastrointestinal side effects, which may occur in up to $20 \%$ of patients treated with diflunisal. Salicylates were provided by OncoAdvance $\mathrm{GmbH}$ (Staufen, Germany). The patients who qualified for such a treatment were in the extreme advanced stage of the disease and had previously undergone multiple treatments. In such cases, the off-label use is justified in Germany. The patients were treated with salicylates only after an extensive informative conversation with the physician and all were required to provide written informed consent.

Metronomic low-dose chemotherapy. One patient received metronomic therapy in combination with the administration of capecitabine and trofosfamide combined with bevacizumab for 61 days. Metronomic low-dose chemotherapy was administered as a long-running daily oral application of cytostatics (capecitabine or trofosfamide) in extremely low doses $\left(1 \mathrm{~g} / \mathrm{m}^{2}\right.$ or $1 \mathrm{~g}$ absolute oral). In 2008, Reichle and Vogt (16) introduced this treatment schedule in combination with anti-inflammatory therapies and angiostatic therapies.

Response assessment by imaging technologies. Tumour response was assessed by PET-CT. The optical evaluation of the response to a therapy followed the Response Evaluation Criteria In Solid Tumors (RECIST) criteria (17). PET was used to visualize the metabolic activity of the tissue. The metabolic assessment of tumour activity was documented, but metabolic activity data were not included in this analysis. The PET-CT
Table I. Patient characteristics $(n=14)$.

Characteristic

Patients, n (\%)

\begin{tabular}{lc}
\hline Gender & \\
Male & $2(14)$ \\
Female & $12(86)$ \\
Localisation of primary & \\
Cervix & $1(7)$ \\
Ovaries & $3(21)$ \\
Breasts & $4(29)$ \\
Endometrium & $1(7)$ \\
Lungs & $2(14)$ \\
Skin & $1(7)$ \\
Orbits & $1(7)$ \\
Unknown & $1(7)$ \\
Metastatic sites & \\
Lymph nodes & $8(57)$ \\
Lung & $7(50)$ \\
Bone & $5(36)$ \\
Brain & $3(21)$ \\
Other & $5(36)$ \\
No. of metastatic sites & \\
0 & $1(7)$ \\
1 & $4(29)$ \\
2 & $4(29)$ \\
\hline
\end{tabular}

was performed by an independent facility, the Radiological Clinic of the University Tübingen (Tübingen, Germany).

RECIST 1.1 in brief. For the RECIST criteria, the number of target lesions is equal to $\leq 2$ per organ, with up to 5 in total. A complete response (CR) is classified as the disappearance of all target lesions. The short axis of any pathological lymph nodes must be reduced to $<10 \mathrm{~mm}$. A partial response (PR) is classified as a $\geq 30 \%$ decrease in the sum of the diameters of the target lesions (SLD), using the baseline sum of diameters as a reference. Progressive disease (PD) is defined as a $\geq 20 \%$ increase in the SLD, using the smallest sum on the study as a reference. The SLD must also show an absolute increase of $\geq 5 \mathrm{~mm}$. Stable disease (SD) is classified as insufficient shrinkage to qualify for PR or an insufficient increase in SLD to qualify for PD.

Quantification of CTCs and tumour cell chemosensitivity test. The quantification of CTCs from the whole blood was performed as previously described (14). In short, red blood cells from $1 \mathrm{ml}$ blood were lysed and the remaining white blood pellet was analysed. Epithelial cells were detected by laser scanning cytometry using the Olympus ScanR screening station (Olympus Corporation, Tokyo, Japan). For staining, the cells were incubated at $4^{\circ} \mathrm{C}$ overnight with a monoclonal fluorescein-isothiocyanate (FITC)-conjugated EpCAM antibody (mouse $\alpha$-human; catalog no. 130-080-301; 1:100; Miltenyi Biotec GmbH, Bergisch Gladbach, Germany). On 
the second day, dead cells were detected with propidium iodide (PI), which is excluded from viable cells but incorporated in dead cells. The baseline CTC assessment for each treatment was performed prior to (maximum, 4 months previously) or during the treatment, and the follow-up assessment was performed at the earliest 2 weeks after the beginning of treatment and between 1 and 6 months after the baseline assessment. A $>5$-fold decrease in CTCs was considered a 'significant decrease', while a $>5$-fold increase was considered a 'significant increase' in CTCs. A $<5$-fold increase or decrease was considered a stable CTC course. The chemosensitivity testing of the patients' CTCs was performed as described previously (18). CTCs among the white blood cells from the whole blood were labelled with the FITC-conjugated EpCAM antibody, as aforementioned. The cytotoxicity of the drug in question was determined by exposing the cells to three different concentrations: The physiological concentration of the drug in the blood of a patient under treatment, plus a 10-fold higher and a 10-fold lower concentration. Dead cells could be distinguished from living cells by PI and EpCAM antibody staining, and subsequent quantification with the laser scanning cytometry. The chemosensitivity rate was calculated as the ratio of dead cells to the total cell number in the sample. The quantification of CTCs from the whole blood and the chemosensitivity testing was performed in the diagnostic laboratory of Dr Ulrich Pachmann (Transfusion Medical Centre Bayreuth, Bayreuth, Germany).

Statistical analysis. All statistical analysis was performed using SigmaPlot software (version 13.0; Systat Software $\mathrm{GmbH}$, Erkrath, Germany). Data was analyzed using Pearson's $\chi^{2}$ test with 4 degrees of freedom. $\mathrm{P}<0.05$ was considered to indicate a statistically significant difference.

\section{Results}

Chemosensitivity of circulating tumour cells. For the present study, 25 patients were screened using the inclusion criteria. For inclusion in the study, the patients had to have at least one chemosensitivity testing, at least one medical treatment followed by PET-CT response assessment and at least two CTC counts, the first one (baseline) shortly prior to or shortly after the beginning of treatment and the second one (follow-up) between 1 and 6 months later. In total, 14 patients met the inclusion criteria. The in vitro sensitivity of the CTCs to salicylates, selected cytostatics and tyrosine kinase inhibitors (TKIs) was tested for each patient individually. Diflunisal was found to be an effective cytotoxic agent in 11 patients (mean chemosensitivity-index, 86\%) and PAS was found to be an effective cytotoxic agent in 8 patients (mean chemosensitivity-index, 73\%), as shown in the results of the in vitro chemosensitivity test (Table II). The cytostatic drugs used in these patients (cisplatin, capecitabine, trofosfamide, paclitaxel, docetaxel and carboplatin) showed high cytotoxic activity ranging from 40 to $95 \%$. The TKIs pazopanib and imatinib also showed cytotoxic activity of $95 \%$ in 2 patients.

Response assessment by PET-CT. The treatment response data of all patients assessed by PET-CT was collected. A
Table II. CTC chemosensitivity assays (performed, n=13).

\begin{tabular}{lc}
\hline Treatment & Mean chemosensitivity rate (patients, $\mathrm{n})$ \\
\hline Diflunisal & $86 \%(11)$ \\
PAS & $73 \%(8)$ \\
Cisplatin & $76 \%(4)$ \\
Capecitabine & $40 \%(4)$ \\
Trofosfamide & $55 \%(4)$ \\
Paclitaxel & $60 \%(1)$ \\
Docetaxel & $95 \%(1)$ \\
Carboplatin & $80 \%(1)$ \\
Pazopanib & $95 \%(1)$ \\
Imatinib & $95 \%(1)$
\end{tabular}

CTC chemosensitivity rate is the ratio of dead cells to the total cell number. This table displays the sensitivity to the drug in physiological concentration. CTC, circulating tumor cells.

total of 21 treatments were assessed (Table III). Additionally, the patient's condition was assessed without previous treatment 6 times (Table III). The median age of the patients was 55.5 years. PET-CTs that were performed no earlier than 2 weeks after the beginning of treatment and no later than 4 months after the treatment were included in the evaluation. CR to a treatment was observed in 1 case after diflunisal treatment. PR to a treatment was observed in 5 cases, three times after treatment with cytostatic drugs in combination with bevacizumab, once after treatment with diflunisal and once after a combined treatment with ASA, tamoxifen and bevacizumab. SD after treatment with diflunisal or other salicylates was observed in 7 cases. SD after treatment with cytostatic drugs was observed in 1 case and SD without previous treatment was observed in 1 case. The objective response rate, which is the sum of the CR and PR rates, was $22 \%$. PD following a treatment was observed in 7 cases and a PD without previous treatment was documented 5 times.

Treatment monitoring by quantification of CTCs. An increase in cell numbers is assumed to correspond to high tumour activity and poor prognosis (13). The results from the present study showed that the behaviour of CTC numbers correlates well with the imaging response. The positive imaging response (CR or $\mathrm{PR}$ ) correlated with a CTC reduction in 5/6 (83\%; $\mathrm{P}=0.030)$ of cases. The assessment of a SD correlated with a stable CTC course in $7 / 9$ cases $(78 \%$; $\mathrm{P}<0.001)$. The assessment of a PD revealed no correlation with an increase in cell numbers in $6 / 12(50 \% ; \mathrm{P}=0.368)$ of cases (Table IV). The $\chi^{2}$ test confirmed that PET-CT outcome and CTC course were significantly correlated $(\mathrm{P}=0.0018)$. Fig. 1 shows three examples of individual treatment courses. CTC quantity was measured during the treatment periods and for follow-up. A patient who was suffering from an endocervical carcinoma started with stable CTC numbers during the initial diflunisal treatment (Fig. 1A). The following decrease from 600 to 0 cells was accompanied by a CR, and the following rise was accompanied by PD. Thereafter, a tamoxifen/bevacizumab treatment resulted in a PR, which was accompanied by stable 
Table III. Response assessment.

\begin{tabular}{|c|c|c|c|}
\hline Patient no. & Treatment & PET-CT & CTCs \\
\hline 1 & Diflunisal & PR & Decrease \\
\hline \multirow[t]{4}{*}{3} & Paclitaxel, Bevacizumab & PR & Decrease \\
\hline & Paclitaxel & PD & Decrease \\
\hline & Doxorubicin, Cyclophosphamide, & & \\
\hline & Bevacizumab & SD & Stable course \\
\hline \multirow[t]{2}{*}{4} & Diflunisal & PD & Decrease \\
\hline & None & PD & Increase \\
\hline \multirow[t]{2}{*}{7} & Diflunisal & SD & Stable course \\
\hline & Diflunisal & SD & Stable course \\
\hline \multirow[t]{3}{*}{8} & Diflunisal & SD & Stable course \\
\hline & None & PD & Stable course \\
\hline & PAS & PD & Decrease \\
\hline \multirow[t]{4}{*}{10} & Diflunisal & SD & Decrease \\
\hline & PAS & SD & Stable course \\
\hline & None & PD & Increase \\
\hline & None & SD & Stable course \\
\hline \multirow[t]{3}{*}{11} & Diflunisal & $\mathrm{CR}$ & Decrease \\
\hline & None & PD & Increase \\
\hline & ASA, bevacizumab, tamoxifen & PR & Stable course \\
\hline 12 & Diflunisal & SD & Stable course \\
\hline \multirow[t]{2}{*}{19} & Diflunisal & PD & Increase \\
\hline & Trofosfamide, Bevacizumab & PR & Decrease \\
\hline 20 & Trofosfamide, bevacizumab, zoledronic acid & PR & Decrease \\
\hline 21 & Diflunisal & SD & Decrease \\
\hline 22 & Gefitinib & PD & Decrease \\
\hline \multirow[t]{2}{*}{23} & Diflunisal & PD & Increase \\
\hline & Diflunisal & PD & Stable course \\
\hline 25 & None & PD & Increase \\
\hline
\end{tabular}

PET-CT, positron emission tomography-computed tomography; ASA, acetylsalicylic acid; PAS, 4-aminosalicylic acid; CTC, circulating tumor cell; CR, complete response; PR, partial response; PD, progressive disease; SD, stable disease.

Table IV. Contingency table PET-CT and CTC course.

\begin{tabular}{|c|c|c|c|c|c|c|c|c|}
\hline \multirow[b]{2}{*}{ PET-CT outcome } & \multicolumn{2}{|c|}{ CTC reduction } & \multicolumn{2}{|c|}{$\begin{array}{c}\text { Stable } \\
\text { CTC course }\end{array}$} & \multicolumn{2}{|c|}{ Increased CTC } & \multirow[b]{2}{*}{ P-value } & \multirow[b]{2}{*}{ Sum, $\mathrm{n}$} \\
\hline & $\mathrm{n}$ & $\chi^{2}$ & $\mathrm{n}$ & $\chi^{2}$ & $\mathrm{n}$ & $\chi^{2}$ & & \\
\hline $\mathrm{CR}+\mathrm{PR}$ & 5 & 2.67 & 1 & 0.67 & 0 & 1.33 & 0.030 & 6 \\
\hline SD & 2 & 0.76 & 7 & 3.33 & 0 & 2.00 & 0.001 & 9 \\
\hline PD & 4 & 0.16 & 2 & 1.34 & 6 & 4.17 & 0.368 & 12 \\
\hline Sum & 11 & & 10 & & 6 & & & 27 \\
\hline
\end{tabular}

$\chi^{2}$ test was performed to 4 degrees of freedom $\left(\chi^{2}\right.$ statistic, $\left.17 ; \mathrm{P}=0.002\right)$. PET-CT, positron emission tomography-computed tomography; $\mathrm{CTC}$, circulating tumor cell; $\mathrm{CR}$, complete response; PR, partial response; $\mathrm{PD}$, progressive disease; $\mathrm{SD}$, stable disease.

CTC numbers (Fig. 1A). The patient in Fig. 1B exhibited rising CTC numbers during the paclitaxel/bevacizumab treatment, but then falling tumour cell numbers indicated successful treatment. Rising cell numbers during paclitaxel treatment have been previously observed and are assumed to originate from enhanced cell dissemination from the primary tumour and most probably also from metastases (19). The treatment success was also reflected by a PR. After the chemotherapy, cell numbers were rising and could be again reduced by paclitaxel therapy. However, 3 months later, PD 

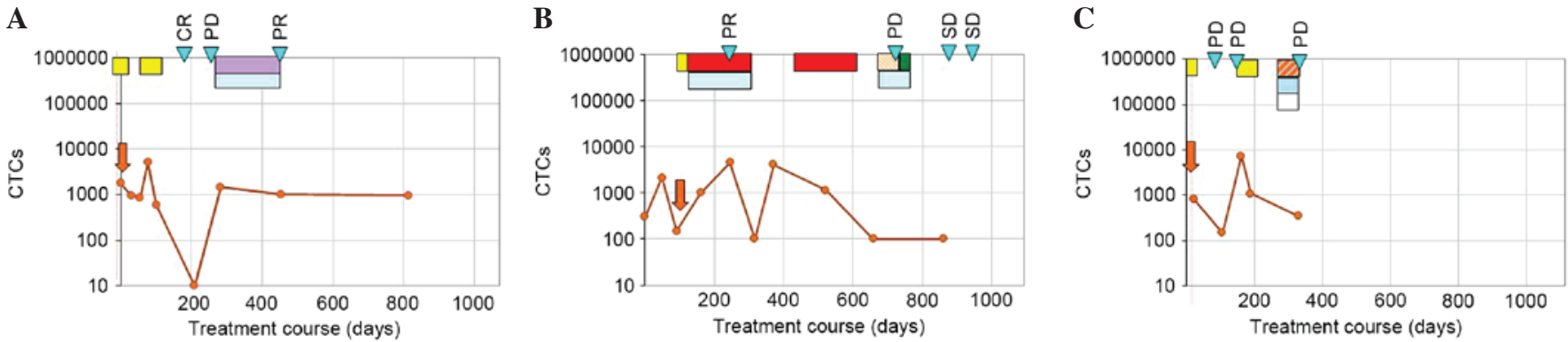

Figure 1. Treatment courses of cancer patients (A-C) Serial CTC assessment: Three patients are shown for whom diagnoses and specific therapies were as follows: (A) Endocervical carcinoma, two treatment periods of diflunisal (yellow bar) followed by tamoxifen/bevacizumab treatment (violet/blue bar); (B) ovarian carcinoma, diflunisal treatment (yellow bar) followed by paclitaxel/bevacizumab and paclitaxel treatments (red and blue bars), doxorubicin/bevacizumab (striped and blue bars) and cyclophosphamide/bevacizumab (green/blue bars); (C) mammary carcinoma, two treatment periods of diflunisal (yellow bar) followed by low-dose capecitabine/trofosfamide/bevacizumab treatment (striped orange/pale blue/white bar). Orange arrows indicate time points for chemosensitivity testing. Blue triangles indicate time points for positron emission tomography-computed tomography. CTC numbers refer to 1 ml blood. CR, complete response; PR, partial response; SD, stable disease; PD, progressive disease; CTC, circulating tumor cell.

was observed despite previously falling numbers. Whether a repeated increase in cell numbers, as had been observed prior to the previous progression, had occurred could not be evaluated, since the next analysis was performed only after an additional treatment. Thereafter a stable CTC course resulting from treatments with doxorubicin/bevacizumab and cyclophosphamide/bevacizumab treatment was reflected by findings of SD in PET-CT. Furthermore, the CTC course of a breast cancer patient (Fig. 1C) showed a decrease in the number of CTCs after a 4-week diflunisal treatment. Subsequent rising CTC numbers were accompanied by PD. Another diflunisal treatment (for 4 weeks) and the following low-dose chemotherapy resulted in a decrease in CTC numbers, while the disease continued to progress, as indicated in Fig. 1C. Again, it was assumed that this was an incomplete response characterized by a positive response on the CTC level, but PD observed by the imaging technique.

The principles outlined in the Declaration of Helsinki and the German Data Protection Act were followed during data collection. Informed consent for the publication of clinical data and CTC count data was obtained from the participants involved.

\section{Discussion}

The majority of patients who seek an integrative treatment at the UNIFONTIS clinic are heavily pre-treated and have already switched from curative to palliative care, or they refuse any treatment with cytostatic agents. The integrative and personalized treatment combines medical treatment with non-medical and naturopathic treatments. The present study assessed the treatment response in 14 patients with advanced epithelial tumours. Treatments were combined treatments with salicylates, cytostatics, bevacizumab, tamoxifen and zoledronic acid.

Overall, 6 patients experienced tumour remission (complete or partial). With respect to the usually poor prognosis of the patients in the palliative situation, this is a reasonable success. The present data confirmed the anticancer effect of salicylates, since disease remission (complete and partial) could be observed after diflunisal treatment. However, further studies with a higher number of cases are required to establish the role of salicylates as anticancer drugs.
It has previously been shown that in cancers, systemic treatment affects CTC numbers and can reduce them $(20,21)$. However, the quantification of CTCs is not generally accepted to be a biomarker for treatment results. The present study provides a notable finding: Positive response assessments by imaging techniques (PR and $\mathrm{CR}$ ) were generally reflected by a reduction in CTC numbers (83\%). Likewise the finding of SD was often accompanied by stable CTC numbers (78\%). However, the PD assessment by PET-CT was not always accompanied by an increase in cell numbers. In the latter case, a reduction of CTCs or stable CTCs could be observed after treatment, while the solid tumour/metastases were clearly growing. This may reflect a response to a medical treatment limited to the CTCs, probably due to insufficient access of the drugs to the respective solid tumour/metastases. The insufficient access is most probably the result of the well-known fact that the majority of tumours exhibit high intra-tumoural pressure, thus creating an interstitial flow in a direction from the tumour to the healthy tissue, which is a major cause of therapeutic failure and treatment resistance (22).

The present study concludes that the analysis of CTC quantities, in addition to the standard procedures for follow-up monitoring, may allow the physician to differentiate between treatments that are clearly not working (no response from the CTCs and a growing tumour mass) and those in which the drugs cannot sufficiently be delivered to the cells inside the tumour mass. This would imply that in this case, actions to reduce the tumour pressure may improve the treatment outcomes and thus provide a more personalized treatment to the patient. The results of the present study are consistent with the findings of $\mathrm{Yu}$ and Cristofanilli (23) who suggested that CTC enumeration may be used to monitor treatment along with imaging techniques. The authors identified a correlation between the two different monitoring modalities in advanced malignancies (23). As a result of the present study, a combined analysis of imaging and CTC quantification appears to be more sensitive than imaging alone and is therefore warranted for the individual treatment monitoring of patients with solid tumours.

\section{Acknowledgements}

The authors would like to thank Ms. Erika Schill and Ms. Laura Redel of the Transfusion Medical Centre Bayreuth 
(Bayreuth, Germany) for providing technical assistance. English language proofreading of the original manuscript was performed by 24translate GmbH (Hamburg, Germany).

\section{References}

1. Thun MJ, Henley SJ and Patrono C: Nonsteroidal Anti-inflammatory drugs as anticancer agents: Machanistic, pharmarcologic and clinical issues. J Natl Cancer Inst 94: 252-266, 2002.

2. Claudius AK, Kankipati CS, Kilari RS, Hassan S, Guest K, Russell ST, Perry CJ, Stark LA and Nicholl ID: Identification of aspirin analogues that repress NF- $\mathrm{KB}$ signalling and demonstrate anti-proliferative activity towards colorectal cancer in vitro and in vivo. Ocol Rep 32: 1670-1680, 2014.

3. Ulrich CM, Bigler J and Potter JD: Non-steroidal anti-inflammatory drugs for cancer prevention: Promise, perils and pharmacogenetics. Nat Rev Cancer 6: 130-140, 2006

4. Retsky M, Rogers R, Demicheli R, Hrushesky WJ, Gukas I, Vaidya JS, Baum M, Forget P, Dekock M and Pachmann K NSAID analgesic ketorolac used perioperatively may suppress early breast cancer relapse: Particular relevance to triple negative subgroup. Breast Cancer Res Treat 134: 881-888, 2012.

5. Kreutz W: Diflunisal for the treatment of cancer. German Patent WO/2005/016354. Filed August 18, 2004. Issued February 24, 2005.

6. Bock JM, Menon SG, Goswami PC, Sinclair LL, Bedford NS, Domann FE and Trask DK: Relative non-steriodal anti-inflammatory drug (NSAID) antiproliferative activity is mediated through p21-induced G1 arrest and E2F inhibition. Mol Carcinog 46 : 857-864, 2007.

7. Rhim AD, Mirek ET, Aiello NM, Maitra A, Bailey JM, McAllister F, Reichert M, Beatty GL, Rustgi AK, Vonderheide RH, et al: EMT and dissemination precede pancreatic tumor formation. Cell 148: 349-361, 2012.

8. Wang RA, Lu YY and Fan DM: Reasons for cancer metastasis: A holistic perspective. Mol Clin Oncol 3: 1199-1202, 2014.

9. Plaks V, Koopman CD and Werb Z: Circulating Tumor Cells. Science 341: 1186-1188, 2013.

10. Gaorav GP and Massagué J: Cancer metastasis: Building a framework. Cell 127: 679-695, 2006.

11. Steeg PS: Tumor metastasis: Mechanistic insights and clinical challenges. Nat Med 12: 895-904, 2002.

12. Budd GT, Cristofanilli M, Ellis MJ, Stopeck A, Borden E, Miller MC, Matera J, Repollet M, Doyle GV, Terstappen LW and Hayes DF: Circulating tumor cells versus imaging-predicting overall survival in metastatic breast cancer. Clin Cancer Res 12: 6403-6409, 2006.
13. Pachmann K, Camara O, Kavallaria A, Krauspe S, Malarski N, Gajda M, Kroll T, Jörke C, Hammer U, Altendorf-Hofmann A, et al: Monitoring the response of circulating epithelial tumor cells to adjuvant chemotherapy in breast cancer allows detection of patients at risk of early relapse. J Clin Oncol 26: 1208-1215, 2008.

14. Pachmann K, Dengler R, Lobodasch K, Fröhlich F, Kroll T, Rengsberger M, Schubert R and Pachmann U: An increase in cell number at completion of therapy may develop as an indicator of early relapse: Quantification of CETC for monitoring of adjuvant therapy in breast cancer. J Cancer Res Clin Oncol 134: 59-65, 2008.

15. Pachmann K, Camara O, Kavallis A, Schneider U, Schünemann S and Höffken K: Quantification of the response of circulating epithelial cells to neoadjuvant treatment for breast cancer: A new tool for treatment monitoring. Breast Cancer Res 7: R975-R979, 2005.

16. Reichle A and Vogt T: Systems Biology: A Therapeutic Target for Tumor Therapy. Cancer Microenviron 1: 159-170, 2008.

17. Eisenhauer EA, Therasse P, Bogaerts J, Schwartz LH, Sargent D, Ford R, Dancey J, Arbuck S, Gwyther S, Mooney M, et al: New response evaluation criteria in solid tumours: Revised RECIST guideline (version1.1). Eur J Cancer 45: 228-247, 2009.

18. Rüdiger N, Stein EL, Schill E, Spitz G, Rabenstein C, Stauch M, Rengsberger M, Runnebaum IB, Pachmann U and Pachmann K: Chemosensitivity testing of circulating epithelial tumor cells (cetc) in vitro: Correlation to in vivo sensitivity and clinical outcome. J Cancer Ther 4: 597-605, 2013.

19. Camara O, Rengsberger M, Egbe A, Koch A, Gajda M, Hammer U, Jörke C, Rabenstein C, Untch M and Pachmann K: The relevance of circulating epithelial tumor cells (CETC) for therapy monitoring during neoadjuvant (primary systemic) chemotherapy in breast cancer. Ann Oncol 18: 1484-1492, 2007.

20. Hekimian K, Meisezahl S, Trompelt K, Rabenstein C and Pachmann K: Epithelial cell dissemination and readhesion: Analysis of factors contributing to metastasis formation in breast cancer. ISRN Oncol 2012: 601810, 2012.

21. Nagrath S, Sequist LV, Maheswaran S, Bell DW, Irimia D, Ulkus L, Smith MR, Kwak EL, Digumarthy S, Muzikansky A, et al: Isolation of rare circulating tumour cells in cancer patients by microchip technology. Nature 450: 1235-1239, 2007.

22. Munson JM and Shieh AC: Interstitial fluid flow in cancer: Implications for disease progression and treatment. Cancer Manag Res 6: 317-328, 2014.

23. Yu JQ and Cristofanilli M: Circulating tumor cells and PET. J Nucl Med 52: 1501-1504, 2011. 